\title{
Management of knee rheumatoid arthritis with total knee arthroplasty: a systematic review
}

\author{
Calin Dragosloveanu, Serban Dragosloveanu, Bogdan Cretu \\ Clinical Hospital for Orthopedics-Traumatology and TB osteoarticular Foisor, Bucharest, Romania
}

\begin{abstract}
Total knee arthroplasty (TKA) in patients with rheumatoid arthritis (RA) has different features from those seen in patients with osteoarthritis. In $197085 \%$ of the patients undergoing TKA had rheumatoid arthritis. Nowadays less than $5 \%$ of the patients undergoing TKA have rheumatoid disease. An important reason for the marked decrease is the improvement of the medical treatment. Fewer patients now progress to permanent structural damage requiring arthroplasty.

TKA in RA has management difficulties that are unique to the disease. These include ipsilateral hip involvement, bilaterality, anticoagulation needs, flexion contractures, rheumatoid cysts and possibly the need for synovectomy. Patients with rheumatoid arthritis are more vulnerable to both early and late infections. They require a prosthesis and technique that provide adequate flexion for activities of daily living. Their often-profound osteopenia can lead to intraoperative and postoperative fractures. Finally, their cervical spine and temporal-mandibular disease create challenging anesthetic considerations. Nevertheless, given these considerations and the degree of preoperative disability suffered by patients with rheumatoid arthritis, the results of surgery can be extremely dramatic and gratifying to both patient and surgeon.
\end{abstract}

Keywords: rheumatoid arthritis, total knee arthroplasty, flexion contractures, rheumatoid cysts, patellar resurfacing

\section{Osteopenia}

The osteopenia associated with rheumatoid disease can present difficulties during TKA. Ruchholtz noted and reported the association of notching the anterior cortex of the femur with postoperative stress fracture in patients with rheumatoid arthritis (1). The risk for stress fracture is sufficiently great in these patients that if the cortex is significantly notched, a long-stemmed femoral component should be considered in these cases. Notching occurs when a femur that is between sizes is prepared for the smaller component.

When osteopenia is severe, supracondylar fractures can occur during preparation of the leg for surgery. In these cases, if the hip above is stiff and the assistant raises the leg with too much force a fracture can occur. Similarly, supracondylar fractures can occur during preparation of the leg for total hip arthroplasty in patients with rheumatoid arthritis, in these cases, too great abduction force applied to the ankylosed hip can produce a fracture in the lower femur.

Fractures also can be generated during postoperative manipulation for poor range of motion after
TKA. This emphasizes the importance of assessing flexion against gravity of the replaced knee at the end of the procedure with the capsule closed so patient's potential is not exceeded.

Another intraoperative potential complication in the patient with severe osteopenia and rheumatoid arthritis is iatrogenic avulsion of the medial collateral ligament from its femoral origin owing to medial retractors. The avulsion usually includes the medial cortex, which separates from the soft underlying cancellous bone. Usually is not a clinical problem, because the soft tissue sleeve remains intact. If it occurs, a cancellous screw with a washer can resecure the cortex to the condyle.

Intraoperative fracture of the patella is also a complication of osteopenia. This might occur after patellar preparation including the placement of three holes for the fixation lugs. These holes are now stress risers, and merely retracting against the patella in a normal fashion can propagate a fracture. Again, the soft tissue sleeve remains intact. If the fragments are minimally separated, the fixation provided by the cemented patellar component across the fracture 
may suffice. If the fragments are separate the surgeon must consider cerclage with wire.

\section{Ipsilateral hip involvement}

In rheumatoid arthritis ipsilateral hip involvement is more frequent than in osteoarthritis. The hip joint should be closely evaluated before TKA, and with some exceptions, the hip should be replaced before TKA (2). There are at least six reasons for this. The first reason refers to reestablishing the anatomical rotation center of the hip before taking into consideration knee surgery. TKA preoperative planning takes into consideration the center of the hip so it makes sense that this should be corrected first.

The second reason stipulates that it is best to first resolve any knee pain that is referred from the hip. Sometimes TKA can be delayed because of the pain relief gained by hip replacement. In some cases it is imperative to resolve the source of the knee pain and the hip joint can be injected with bupivacaine under fluoroscopic control. The patient can then report on the extent of pain relief gained. If the pain relief is considerable, both patient and surgeon are more comfortable with the decision to proceed with the hip first.

The third reason is important for the juvenile patient with rheumatoid arthritis. Because the hip surgery is relatively easy and painless for the patient (compared with TKA), the surgeon gains the patient's confidence. If the knee is operated first, the pain and difficult rehabilitation that the patient endures without a significant gain in function discourages the patient who still has pain and lack of function.

A fourth related reason is the fact that a person can exercise a hip above a painful knee, whereas it is difficult to exercise a knee below a painful and stiff arthritic hip. A stationary bicycle is extremely helpful during rehabilitation of total knee arthroplasty but is not important to rehabilitation of a hip replacement. The use of a bicycle is not possible when the hip above is painful and stiff.

The fifth reason is to resolve the tension of muscles that cross both the hip and knee joint, especially the hamstrings. If both the hip and the knee have flexion contractures and the knee is operated on first, resolving that contracture, a subsequent hip replacement that lengthens the hip can retighten the hamstring muscle.

The sixth reason also is related to preoperative knee contractures. At the time of hip arthroplasty, a contracted knee can be manipulated and casted to improve passive extension before the knee replacement. If epidural anesthesia is used, it can be maintained for several days and serial casts can be applied.

Seventh, it makes sense to avoid twisting and torqueing a well-balanced TKA while dislocating and exposing a stiff hip for replacement.

\section{Flexion contracture}

Flexion contractures are more frequently seen in rheumatoid arthritis than in normal osteoarthritis (Fig. 1). The contracture is caused by the inflammation in the soft tissues, whereas the flexion contracture in normal osteoarthritis is associated with bony block (Fig. 2). After studying the outcomes of a large number of patients with severe contractures Scott developed the following treatment guidelines for patients with rheumatoid arthritis (3).

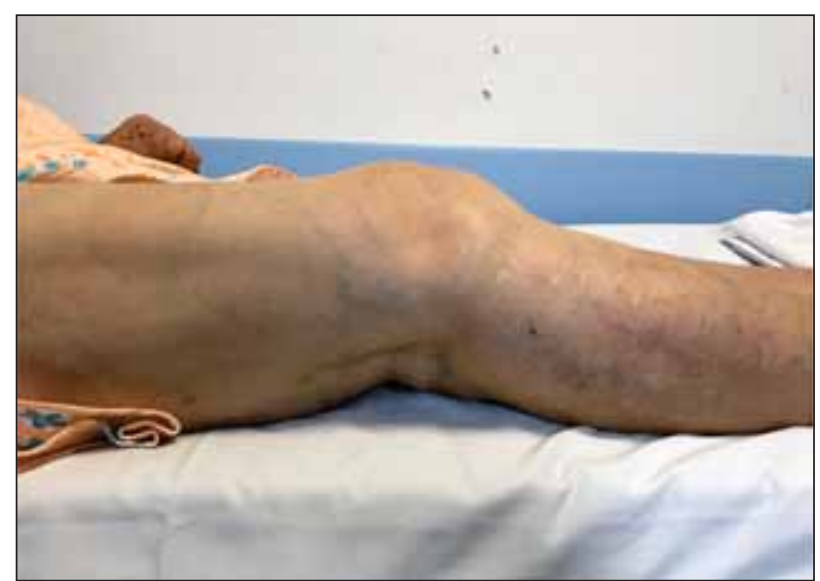

FIGURE 1

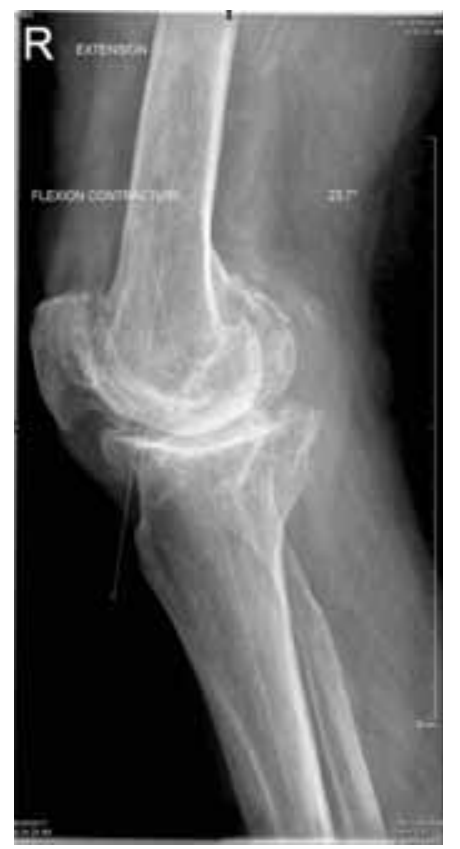

FIGURE 2 
For flexion contractures less than 15 degrees after anesthesia, distal femoral resection and posterior capsular is done in a normal fashion. If the flexion contracture is between 15 and 45 degrees, distal femoral resection is increased by $2 \mathrm{~mm}$ for every additional 15 degrees of correction that is necessary. This technique has a limit of a total of $13 \mathrm{~mm}$ to avoid compromising the femoral origins of the collateral ligaments.

Between 45 and 60 degrees of flexion contracture, preoperative manipulation and casting has to be done and always is indicated to use a posterior cruciate ligament (PCL)-substituting technique. For flexion contractures greater than 60 degrees consider preoperative manipulation and casting as well and a constrained prosthesis to resolve flexion gap laxity that can result from significant elevation of the femoral joint line.

For patients with rheumatoid arthritis it is indicated to follow the ,rule of one third“. This states that intraoperative correction needs only to be within one third of the preoperative contracture under anesthesia. The residual one third usually resolves with physical therapy, resolution of the inflammatory disease, and occasionally the help of manipulation and casting.

Several additional points regarding flexion contractures should be discussed. If the patient has severe bilateral flexion contractures, both should be corrected simultaneously to avoid the risk for regression of the correction on the first side until the second side is corrected.

When the capsule is closed after correction of a severe flexion contracture, the medial capsule should be advanced distally on the lateral capsule to avoid an initial extensor lag.

The amount of posterior slope applied when dealing with a severe flexion contracture should be zero degrees. Every degree of slope built into the tibia creates a degree of flexion contracture it prevents its correction.

Finally, preventive measures should be taken after surgery, such as allowing the patient to have a roll under the ankle but never under the knee. A knee immobilizer at night will prevent the patient sleeping with flexed knees and redeveloping a contracture. A dynamic extension splint can be helpful to both correct and maintain extension in refractory cases.

\section{Synovectomy and recurrent active rheumatoid synovitis}

As mentioned earlier, it is possible for active rheumatoid synovitis to recur after TKA. The potential is increased if the patella has not been resurfaced and residual cartilage remains in the knee joint.

Even with TKA that includes patellar resurfacing there are cases when the rheumatoid synovitis reappears. These patients present a diagnostic problem. They have an acute presentation with a large effusion, an elevated sedimentation rate, and an aspirate with a high cell count. In this scenario, infection is the most likely possibility, but active rheumatoid arthritis also must be considered. The cell count can be as high as 20,000 or 30,000 white cells per high-power field with a predominance of either polymorphonuclear cells or lymphocytes. The percentage of polymorphonuclear cells will not be in the high 90 s as would be characteristic of infection. Cultures will be negative. Percutaneous synovial biopsy for histology may be necessary to confirm the diagnosis of rheumatoid disease. Usually this occurs in the presence of a rheumatoid flare with multiple joint involvements. Appropriate medical treatment is initiated. Occasionally, open synovectomy may be required.

This raises the question of whether synovectomy should be performed at the time of primary arthroplasty in the rheumatoid knee (4). We recommend that this be done universally in patients with active synovitis at the time of the TKA. If the disease is quiet and the synovium uninflamed, synovectomy probably is not necessary.

\section{Rheumathoid cysts}

Small juxtaarticular cysts are common in osteoarthritis but they are more common in rheumatoid arthritis, and sometimes very large cysts are present on either femoral or tibial side of the joint. Sometimes cysts are found after the preliminary standard bone cuts of the femur or tibia (Fig. 3). All cysts should always be curetted free of soft tissue and grafted with cancellous bone to seal the cyst wall from the cement interface. Failure to do this in patients with rheumatoid arthritis leads to the possibility of progressive demarcation at the cement-cyst interface, leading to component loosening. Examination of the tissue at the loosening interface shows histologic features consistent with those of recurrent rheumatoid synovium (5). 


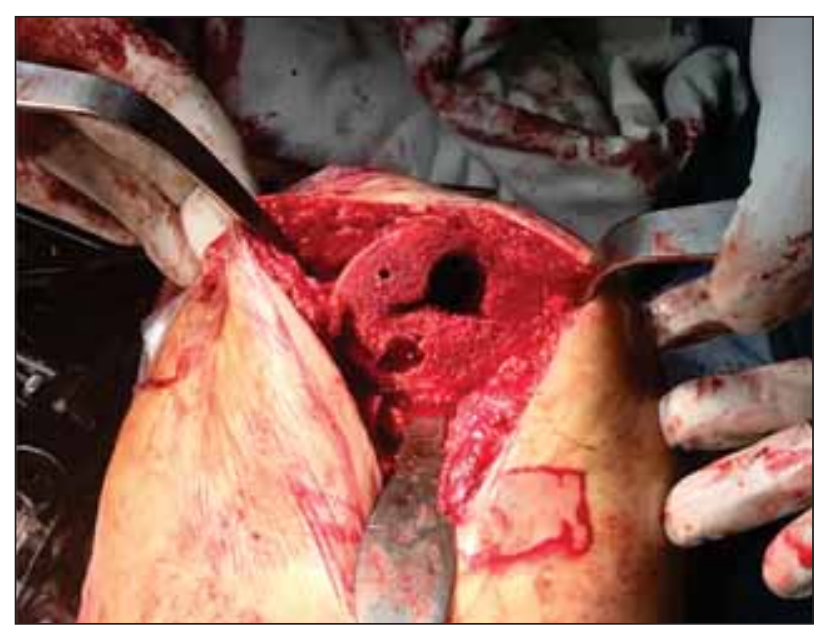

FIGURE 3

Larger central defects can be treated with the impaction grafting technique. In this technique morselized bone graft is packed densely around a long trial stem.

When the trial is removed, the graft should be so densely packed as to provide structural integrity. If the cemented technique is chosen, the canal is plugged with a cement restrictor before application of the bone graft.

\section{Risk for infection}

The risk for perioperative and late infection is greater in patients with rheumatoid arthritis than in those with osteoarthritis. An acceptable rate is around $0.5 \%$ (6). Late infection will occur at an increased frequency owing to the immunosuppressed state of these patients resulting from disease or from medications and because they often have multiple remote sites of chronic infection that can metastasize to the arthroplasty site. The most frequent sites are in foot and lower leg. The olecranon bursa is another possible site.

\section{Patellar resurfacing}

Patellar resurfacing during TKA remains debatable. From the author experience short-term and longterm results in patients with classic arthritis treated with TKA show no difference between patellar resurfacing and without patellar resurfacing. Some studies indicate that for rheumatoid arthritis longterm results are better for patients when the patella was resurfaced (7). Sledge indicated that residual cartilage in the knee of patients with rheumatoid arthritis after TKA was a factor that allowed synovitis to recur (8).

\section{Adequate flexion}

Patients with rheumatoid arthritis require more knee flexion than those with osteoarthritis to have satisfactory function. All people need 60 to 70 degrees of flexion to walk on level ground. Ninety degrees of flexion is necessary for ascending most stairs. One hundred degrees of flexion is needed to descend stairs in a normal fashion. One hundred five degrees or more is necessary to rise from a standard chair without pushing up using the upper extremities.

The frequent involvement of shoulders, elbows, wrists, and hands in patients with rheumatoid arthritis compromises their ability to ascend stairs or rise from a sitting position if they have inadequate knee flexion. Their upper extremity involvement also may require them to use a platform crutch or walker that cradles the forearm with vertical handles for grips. A platform crutch can usually be modified for use by even the most severely involved patients.

\section{Anticoagulation}

Deep vein thrombosis (DVT) and pulmonary emboli occur less frequently in patients with rheumatoid arthritis than in those with osteoarthritis. This may be partially due to the fact that most patients with rheumatoid arthritis require the chronic use of antiinflammatory medications, which have a mild anticoagulation effect. It might also be intrinsically related to their disease process. The reason is unclear, but it makes their anticoagulation needs different from those of patients with osteoarthritis.

In recent years, there has been significant progress toward developing more effective and practical tromboprophylaxis agents to include either low molecular weight heparin or the latest anticoagulant agents (factor Xa inhibitors and direct thrombin inhibitors) (9-11). Chemical prophylaxis agents included in the clinical guidelines were aspirin, warfarin, LMWH, fondaparinux, dabigatran, rivaroxaban, and apixaban.

The American College of Chest Physicians (ACCP) guidelines recommended aspirin as a chemoprophylaxis agent. This is due to the Pulmonary Embolism Prevention (PEP) trial, which concluded that low-dose aspirin, when taken for 35 days, would result in 7 times less symptomatic DVT cases, but in 3 bleeding cases and 2 nonfatal myocardial infarction per 1,000 patients $(12,13)$. 


\section{Anesthetic considerations}

Because of rheumatoid involvement of the cervical spine and temporomandibular joint, special considerations often are required to anesthetize the patient with rheumatoid arthritis. Preoperative assessment by the anesthesiologist is extremely important to anticipate the individual needs of the patient. Lateral cervical spine films in maximum flexion and extension may be appropriate to evaluate the patient for $\mathrm{C} 1-\mathrm{C} 2$ subluxation. Because of the many potential problems associated with the administration of general anesthesia, regional anesthesia is preferred. It can be by either the spinal or epidural route. Still, the surgeon must be prepared for the possible need for general anesthesia and intubation. The anesthesiologist should be skilled in the use of a pediatric fiberoptic laryngoscope and awake nasotracheal intubation techniques.

\section{Posterior cruciate ligament preservation versus substitution}

The literature is controversial regarding the need for PCL substitution versus the acceptability of PCL preservation. Some articles favor PCL substitution while others show good results at 10 years with PCL preservation (14-16). There are two hypotheses to explain the discrepancy in results. In the PCL-sparing technique, knees were implanted in an era when some laxity in the articulation was favored. The ligaments in many patients with rheumatoid arthritis have tendency to stretch out over time, leading to hyperextension, instability, and synovitis. Also during that era, an undersized inset patella often was used. This allowed for retention of residual cartilage in the knee, which provided an increased risk for active rheumatoid synovitis to recur in the knee, along with its adverse consequences.

Conflict of interest: none declared Financial support: none declared

\section{REFERENCES}

1. Ruchholtz S., Tomas J., Gebhard F., Larsen M.S. Periprosthetic fractures around the knee - the best way of treatment. Eur Orthop Traumatol. 2013 Jun; 4(2): 93-102.

2. Singh J.A., O'Byrne M.M., Colligan R.C., Lewallen D.G. Pessimistic explanatory style: a psychological risk factor for poor pain and functional outcomes two years after knee replacement. J Bone Joint Surg Br. 2010, 92: 799-806.

3. Yan D, Yang J, Pei F. Total knee arthroplasty treatment of rheumatoid arthritis with severe versus moderate flexion contracture. J Orthop Surg Res. 2013; 8:41.

4. Hernigou P., Flouzat Lachaniette C.H. „Is Synovectomy Necessary in Total Knee Arthroplasty with Rheumatoid Arthritis?" March 1, 2016 presentation at the annual meeting of the American Academy of Orthopaedic Surgeons.

5. Vasudevan A., DiCarlo E.F., Wright T., Chen D., Figgie M.P., Goldring S.R., MandI L.A. Cellular response to prosthetic wear debris differs in patients with and without rheumatoid arthritis. Arthritis Rheum. 2012; 64:1005-1014.

6. Parvizi J., Adeli B., Zmistowski B., Restrepo C., Greenwald A.S. Management of periprosthetic joint infection: the current knowledge: AAOS exhibit selection. J Bone Joint Surg Am. 2012; 94(14):e104.

7. Barrack R.L., Wolfe M.W. Patellar resurfacing in total knee arthroplasty. J Am Acad Orthop Surg. 2000; 8:75-82.

8. Steinberg J., Sledge C.B., Noble J. et al. A tissue-culture model of cartilage breakdown in rheumatoid arthritis: quantitative aspects of proteoglycan release. Biochem J 1979; 180:403-412.

9. Jameson S.S., Charman S.C., Gregg P.J., Reed M.R., van der Meulen J.H. The effect of aspirin and low-molecular-weight heparin on venous thromboembolism after hip replacement: a non-randomized comparison from information in the National Joint Registry. J Bone Joint Surg (Br) 2011; 93-B:1465-70.

10. Eriksson B.I., Kakkar A.K., Turpie A.G. et al. Oral rivaroxaban for the prevention of symptomatic venous thromboembolism after elective hip and knee replacement. J Bone Joint Surg (Br) 2009; 91-B:636-44.

11. Raskob G.E., Gallus A.S., Pineo G.F. et al. Apixaban vs enoxaparin for thromboprophylaxis after hip or knee replacement: pooled analysis of major venous thromboembolism and bleeding in 8464 patients from the ADVANCE-2 and ADVANCE-3 trials. J Bone Joint Surg (Br) 2012; 94-B:257-64.

12. Geerts W.H., Pineo G.F., Heit J.A. et al. Prevention of venous thromboembolism: the Seventh ACCP Conference on Antithrombotic and Thrombolytic Therapy. Chest. 2004; 126(Suppl):338S-400.

13. Prevention of pulmonary embolism and deep vein thrombosis with low dose aspirin: Pulmonary Embolism Prevention (PEP) trial. Lancet. 2000; 355:1295-302.

14. Marczak D., Kowalczewski J. et al. An evaluation of the posterior cruciate ligament function in total knee arthroplasty with regard to its morphology and clinical properties. Folia Morphol (Warsz). 2017; 76(1):94-99.

15. Tang W.M., Chiu K.Y., Ng T.P., Yau W.P. Posterior cruciate ligament-substituting total knee arthroplasty in young rheumatoid patients with advanced knee involvement. J Arthroplasty. 2004 Jan; 19(1):49-55.

16. Schai P.A., Scott R.D., Thornhill T.S. Total knee arthroplasty with posterior cruciate retention in patients with rheumatoid arthritis. Clin Orthop 1999; 367:96-106. 